\title{
Modelling the Population Dynamics of Root Hemiparasitic Plants Along a Productivity Gradient
}

\author{
Pavel Fibich • Jan Lepš • Luděk Berec
}

(C) Institute of Botany, Academy of Sciences of the Czech Republic 2010

\begin{abstract}
Root hemiparasitic plants interact with their host plants through parasitism and competition. The interactions can be divided into aboveground and belowground interactions. Because both groups of plants are autotrophic, they compete for light aboveground. Belowground interactions are more complex. The host plants compete for resources in the soil and the hemiparasitic plants prey on the host plants through haustoria, using the hosts as the main source of water and nutrients. In this paper, we modeled the relationship between these two plant types, extending the well-known Rosenzweig-MacArthur predator-prey model to cover both light competition and intra-specific parasitism among hemiparasites. We included a realistic relationship of carrying capacity to environmental productivity and followed model behavior on a productivity gradient. The model shows that, at very low productivities, there are only a few poor hosts and hemiparasites have no chance to persist. As productivity increases, there is a range of productivity where both plant types coexist. A further increase in productivity gets the system out of the coexistence range, and only host plants survive. This final prediction successfully explains patterns observed in empirical data, contrary to the results of an earlier, oversimplified model of the explored interaction. Comparison of various models demonstrates that the model is able to reproduce the decline of hemiparasites with increasing productivity only when competition for light is included.
\end{abstract}

Keywords Hemiparasites · Light competition · Productivity gradient ·

Rosenzweig-MacArthur model

P. Fibich $(\bowtie) \cdot$ J. Lepš

Department of Botany, Faculty of Science, University of South Bohemia, Branišovská 31,

CZ-370 05 České Budějovice, Czech Republic

e-mail: pavel.fibich@prf.jcu.cz

J. Lepš $\cdot$ L. Berec

Institute of Entomology, Biology Centre of the Academy of Sciences of the Czech Republic,

Branišovská 31, CZ-370 05 České Budějovice, Czech Republic

L. Berec

Institute of Mathematics and Biomathematics, Faculty of Science, University of South Bohemia,

Branišovská 31, CZ-370 05 České Budějovice, Czech Republic 


\section{Introduction}

Root hemiparasitic plants (e.g., Rhinanthoidae in Orobanchaceae) are parasitic belowground, obtaining most of their water and nutrients from their hosts, and simultaneously autotrophic, producing carbohydrates by photosynthesis (Press 1989; Cameron et al. 2005; Press and Phoenix 2005). They are usually generalists and can potentially attack a large number of different co-occurring plant species simultaneously, yet some host species are preferred (Matthies 1996; Svensson and Carlsson 2004; Press and Phoenix 2005) and some hosts defend themselves better than others (Cameron et al. 2006, 2008). Potential hosts are often perennial grasses, because they are common components of grassland plant communities and have a dense root system (Seel and Press 1996; Puustinen and Salonen 1999), but hemiparasites might also parasitize on each other (Prati et al. 1997). Most root hemiparasites are obligate parasites, but many of them can survive to seedling stage without any host plant (Press 1989; Press and Phoenix 2005). Hemiparasitic plants interact with their hosts both aboveground and belowground and abiotic factors modify these interactions (Cameron et al. 2005). Aboveground, both groups of species compete for light. The belowground interactions are more complex. Both groups of plants potentially compete for soil resources. However, for hemiparasites this interaction is not very important, because they prey on host plants through haustoria attached to the host roots, using them as the main source of water and nutrients (Press and Phoenix 2005; Ahonen et al. 2006). Also, they are hardly able to decrease the level of soil resources by direct uptake to negatively affect the hosts. Resource parasitism allows root hemiparasites to overcome deficits in their competitive abilities (Smith 2000).

Matthies (1995) suggested that hemiparasites are restricted to nutrient-poor habitats, because of the balance between the effects of parasitism and light competition. In highproductivity environments, increased shading may reduce competitiveness of these autotrophic plants with respect to their host plants, which are usually better competitors for light (Press and Phoenix 2005). The importance of aboveground competition grows with increasing productivity of the environment (Grime 1979; Tilman 1988; Wilson and Tilman 1993; Lepš 1999). Also, seedling recruitment of hemiparasitic species might be suppressed within dense vegetation (Petrů and Lepš 2000).

Smith (2000) proposed a simple model of the root hemiparasite - host plant system, assuming that the belowground interactions are much stronger than the aboveground ones and thus neglecting the latter. His model also implicitly assumed that the hemiparasitic plants could complete their life cycle without host plants and is therefore suitable only for facultative hemiparasites. The form of trophic function used in Smith's (2000) model implies that sufficiently abundant nutrients in soil would fully substitute the host. However, this is not the case for obligate hemiparasites, and rarely for facultative hemiparasites. The model thus produced some unrealistic results. First, it predicted that, with increasing productivity, the proportion of biomass of hemiparasitic plants should also increase. However, this prediction contradicts most field observations (e.g., Matthies 1995). Second, the hemiparasites were predicted to outcompete their hosts in environments with high resource levels, or at least increase their representation with increasing level of resources (depending on model parameters).

Cameron et al. (2009) developed a simple model of hemiparasite-grass-forb interactions, parameterizing it with data from pair-wise interaction experiments under 
two nutrient levels between the hemiparasite and several grass and forb species. Whereas stable dynamics (all three plant types present) were observed to emerge under low-nutrient levels, high-nutrient conditions produced unstable dynamics (hemiparasites absent); the latter can be stabilized by considering spatially explicit models (Cameron et al. 2009). Although invaluable in showing that hemiparasites can modify competitive interactions among their hosts and as a consequence cause shifts in host community structure, Cameron et al.'s (2009) model is mainly statistical (fitted to a specific system at two selected nutrient levels), and not aiming explicitly to distinguish the aboveground and belowground interactions between species. In our opinion, the distinction between aboveground and belowground interactions is the key to understanding changes in the behavior of the system along a productivity gradient, and so we attempted to build a model that separates their effects.

In this article, we propose a new model of the root hemiparasite-host plant interaction that removes the two unrealistic assumptions made by Smith (2000) and thus aims at explaining field observations. Namely, we assume intra-specific parasitism among hemiparasites and modify the functional response to cover the aboveground competition for light. Biological assumptions forms a sort of type IV functional response (Freedman and Wolkowicz 1986; Kot 2001). Because our results are opposite to those of the Smith (2000) model, we compare our predictions of system dependence on environmental productivity with a relationship that we were able to extract from published data. By comparing various models, we address which processes must be included in the model to successfully explain and understand the emerging empirical pattern.

\section{Population Dynamics Model}

To develop our model of the hemiparasite-host interaction, we started with the wellknown Rosenzweig-MacArthur predator-prey model, based on the logistic growth of prey and the type II functional and numerical responses of predators (Berryman 1992; Kot 2001; Pastor 2008):

$$
\begin{aligned}
& \frac{\mathrm{d} x}{\mathrm{~d} t}=r x\left(1-\frac{x}{K}\right)-f(x) y \\
& \frac{\mathrm{d} y}{\mathrm{~d} t}=-m y+e f(x) y
\end{aligned}
$$

Here $x$ and $y$ denote prey and predator densities, respectively, $r$ is the intrinsic prey growth rate and $K$ their carrying capacity, both in the absence of predation, $m$ is the per capita mortality rate of predators in the absence of prey, $f(x)$ is the type II functional response and $e$ is an efficiency with which consumed prey are transformed into new predators.

Our prey is a host plant and our predator is a hemiparasitic plant. Mortality parameter $m$ reflects a decrease of biomass of hemiparasitic plants, not death of individuals. Consequently, hosts are not directly consumed. Nevertheless, the amount of water and nutrients present in the host and available to the hemiparasite is proportional to the host biomass, and hemiparasites' use of water and nutrients effectively decreases the biomass of the host. Therefore, we consider $x$ and $y$ as host and hemiparasite biomass 
(not densities), respectively. Consequently, the relationship between biomass and water and nutrients has a similar functional form: thus the parameter $e$ in our case describes an efficiency of hemiparasites' use of water and nutrients from the host biomass to build its own biomass. If the right-hand side of the host equation in (1) is negative, the effect of hemiparasites is so strong that photosynthesis is not able to replace biomass losses due to respiration and/or dying off of the green tissue. The type II functional response is assumed here to have a hyperbolic form

$$
f(x)=\frac{a x}{x+b}
$$

where $a$ is the maximum hemiparasite per capita "predation" rate and $b$ is the host biomass necessary to achieve one-half that of the maximum rate (Abrams and Ginzburg 2000; Kuang 2004; Ellner and Guckenheimer 2006).

We extended model (1) to cover competition for light and intra-specific parasitism among hemiparasites to get the following form:

$$
\begin{aligned}
& \frac{\mathrm{d} x}{\mathrm{~d} t}=r x\left(1-\frac{x+c y}{K}\right)-f(x) y \\
& \frac{\mathrm{d} y}{\mathrm{~d} t}=-\left(m+m_{1} y\right) y+e f(x) y g(x)
\end{aligned}
$$

In this model, $c$ denotes the competitive ability of hemiparasites for light relative to their hosts, $m_{1}$ is a measure of negative interactions (density dependence) among hemiparasites, and $g(x)$ is a function that scales light availability. Negative interactions among hemiparasites come from two sources: getting nutrients from each other (intra-specific parasitism) and shading each other (Prati et al. 1997; Matthies 2003). The scaling function $g(x)$ reflects that with increasing host biomass there is a decreasing amount of light available to hemiparasites. In high density (and so large-biomass) host populations, there is not enough light for hemiparasites that are also autotrophic (Press 1989; Westbury and Dunnett 2007). Therefore, the scaling function $g(x)$ is assumed to decrease with host biomass; for the sake of simplicity, we assume it declines sigmoidally from 1 to 0 as follows:

$$
g(x)=\frac{d^{2}}{x^{2}+d^{2}}
$$

where $d$ is the host biomass necessary to achieve the scale of one-half.

Model (2) thus describes interactions between hosts and hemiparasites due to two limiting resources, light and one general soil resource (nutrients and water together). Both resources are gained from a common resource pool. The host equation defines the logistic growth of host plants with aboveground competition for light, belowground competition for resources, and parasitism. Host plants will grow to the carrying capacity of the environment without hemiparasitic plants. The hemiparasite equation describes hemiparasite intra-specific parasitism and their composite numerical response. This composite response is given by a hyperbolic type II response that is scaled by light availability via Eq. 3, together forming a sort of type IV unimodal response.

All the parameters in our model (2) are positive. We also assume that $b<d$, which means that the half-saturation of hemiparasite consumption (parameter $b$ ) is realized at a smaller host biomass than half-saturation of shading by hosts (parameter $d$ ). 
We could simplify model (2) by omitting parameter $r$, the intrinsic per capita host growth rate, through linearly rescaling time and making appropriate transformations in relevant parameters:

$$
\bar{t}=r t ; \bar{m}=m / r ; \bar{m}_{1}=m_{1} / r ; \bar{a}=a / r
$$

The new and final system to be analyzed is obtained by these substitutions, bars dropped:

$$
\begin{aligned}
& \frac{\mathrm{d} x}{\mathrm{~d} t}=x\left(1-\frac{x+c y}{K}\right)-\frac{a x}{x+b} y \\
& \frac{\mathrm{d} y}{\mathrm{~d} t}=-\left(m+m_{1} y\right) y+e \frac{a x}{x+b} y \frac{d^{2}}{x^{2}+d^{2}}
\end{aligned}
$$

The carrying capacity is driven by a combination of both the limiting resources (light, water and nutrients) in the environment. Changing productivity of the environment leads to a change in the carrying capacity of host plants. We assume that there is a maximum carrying capacity of host plants such that any further increase in productivity does not increase the carrying capacity. This assumption reflects that the carrying capacity of host plants directly follows the productivity gradient until reaching the state where the plants are albeit not limited by nutrients, but rather biomass production reaches other limits (such as body structure or other abiotic factors such as space). Host plants have often wider ecological amplitudes that span even more productive environments. So, we could assume that all the interesting changes in the interactions between hosts and hemiparasites occur in the range where the carrying capacity of the host population is determined by productivity of the environment.

\section{Results}

We will follow model (4) along the productivity gradient and show possible scenarios with respect to productivity or equivalently the host carrying capacity $K$. Equilibrium points of model (4) occur at the intersections of host and hemiparasite isoclines. We are going to identify these points along the productivity gradient (or $K$ ) and determine their stability. Most of our results are based on numerical calculations using Matlab with Matcont package for analysis of equilibria (Dhooge et al. 2003) and Pplane package for visualization of isoclines and dynamics (Polking and Arnold 1999).

\section{Isoclines}

By setting the right-hand sides of Eq. 4 to zero, we obtain the zero-growth isoclines; their intersections determine equilibrium points of the system. Schematic forms of possible patterns of the relative position of host and hemiparasite plant isoclines are sketched in Fig. 1.

The host equation has two isoclines. One is trivial: $x=0$, the hemiparasite axis (i.e., the complete absence of the host, which is biologically uninteresting). The second isocline is

$$
h(x, K)=y=\frac{-x^{2}-b x+(x+b) K}{x c+b c+a K}
$$


Fig. 1 Generalized phase planes of system (4) along increasing productivity with isoclines specified by the hemiparasite equation $p$ (solid line) and the host equation $h$ (dotted line). The points $A$ and $B$ are intersections of the hemiparasite isocline with the $x$ (host) axis and $K$ is the carrying capacity of the host plants. Filled circles denote the stable equilibrium point with host dominance and no hemiparasites. Empty circles (E1 and E3) mark points that are either stable or unstable coexistence equilibria. Squares (E2 and $K$ ) denote unstable saddle points. The following parameters of Eq. 4 could be used to draw similar types of phase planes: $e=1 ; a=0.6$; $b=6 ; d=8 ; m=0.1 ; m_{1}=0.01$; $c=0.1$ and for $K \mathbf{a}-\mathbf{e}: 0.8,6$, 14, 18 and 23
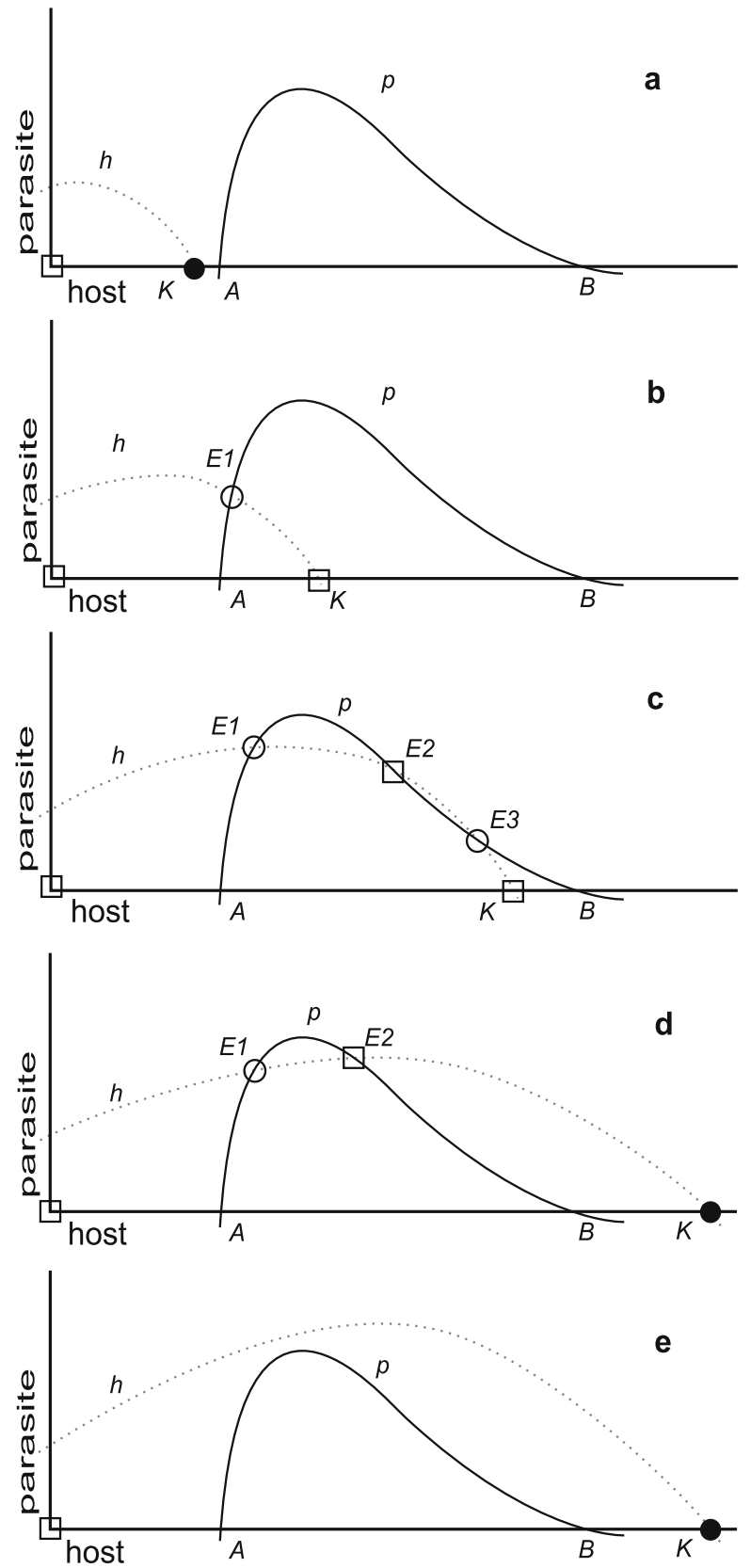

and intersects the $x$ (host) axis at $x=K$ and the $y$ (hemiparasite) axis at

$$
h(0, K)=y=\frac{b K}{b c+a K}
$$

The intersection with the $y$ axis defines a hyperbolic function with respect to $K$ and increases with increasing $K$ to the maximum value $y=b / a$. 
The hemiparasite equation also has two isoclines. The first is trivial: $y=0$ or the host axis. The second is then

$$
p(x)=y=\left(\frac{x a d^{2} e}{(x+b)\left(x^{2}+d^{2}\right)}-m\right) / m_{1}
$$

and could intersect the $x$ axis at three points, but at most two of them are positive. If there are no positive intersections, then hemiparasites decline and host plants grow to the carrying capacity. We focus only on parameter ranges that lead to two positive intersections of $p(x)$ with the $x$ axis. These points are hard to express analytically and we denote them as $A$ and $B$, supposing $A<B$. We see that the hemiparasite isocline does not depend on the carrying capacity $K$ and thus does not change when we increase environmental productivity.

\section{Trivial System Equilibria}

By trivial system equilibria we mean those in which one or both species do not persist. There are two trivial equilibria of model (4), $[0,0]$ and $[K, 0]$, and both always exist. The extinction equilibrium $[0,0]$ describes the situation with no host plants and no hemiparasites. As the determinant of the Jacobian (for the Jacobian of model (4), see Appendix 1) evaluated at this equilibrium

$$
\operatorname{det}\left(\boldsymbol{J}\left[\begin{array}{l}
0 \\
0
\end{array}\right]\right)=-m
$$

is always negative, $[0,0]$ is an unstable equilibrium (saddle point) (the RouthHurwitz criterion; Kot 2001).

The host-only equilibrium $[K, 0]$ describes full dominance of hosts with their biomass at the carrying capacity and no hemiparasites. The determinant and trace of the Jacobian evaluated at this equilibrium are as follows:

$$
\begin{aligned}
\operatorname{det}\left(\boldsymbol{J}\left[\begin{array}{l}
K \\
0
\end{array}\right]\right) & =m-\frac{a d^{2} e K}{(K+b)\left(K^{2}+d^{2}\right)} \\
\operatorname{trace}\left(\mathbf{J}\left[\begin{array}{l}
K \\
0
\end{array}\right]\right) & =\frac{a d^{2} e K}{(K+b)\left(K^{2}+d^{2}\right)}-m-1
\end{aligned}
$$

By applying the Routh-Hurwitz criterion, the equilibrium is unstable (saddle point) when the determinant is negative and stable if the determinant is positive and the trace is negative. You may notice that the determinant and the trace can be expressed using the hemiparasite isocline as

$$
\begin{aligned}
\operatorname{det}\left(\mathbf{J}\left[\begin{array}{c}
K \\
0
\end{array}\right]\right) & =-p(K) m_{1} \\
\operatorname{trace}\left(\mathbf{J}\left[\begin{array}{c}
K \\
0
\end{array}\right]\right) & =p(K) m_{1}-1
\end{aligned}
$$

This implies that if the carrying capacity $K$ is between $A$ and $B$, then the determinant is negative, because $p(x)$ is positive for any $x$ in the range $(A, B)$. In such a case, the equilibrium $[K, 0]$ is a saddle point (Fig. $1 \mathrm{~b}$ and c). If $K$ lies outside the range $(A, B)$, 
then the determinant is positive and the trace is negative, as $p(K)<0$ in this case: hence, $[K, 0]$ is a stable equilibrium (Fig. 1a,d and e).

\section{Non-trivial System Equilibria}

By non-trivial system equilibria we mean those in which both host and hemiparasite plants coexist. Because of the high complexity of Eq. 4, the following results for non-trivial equilibria are based on numerical calculations and show the possible types of equilibria that can occur.

\section{Carrying Capacity K Lies Outside (A,B) Range}

If $K<A$, then there are only two trivial equilibria $[0,0]$ and $[K, 0]$. We know from above that only the second one is stable (see Fig. 1a). The carrying capacity of host plants is too low and hosts attain too low a biomass to sustain hemiparasites. Therefore, host plants grow to the carrying capacity $K$ and hemiparasites decline to extinction.

If $K>B$, then there are two possibilities: either the host isocline $h$ intersects the hemiparasite isocline $p$ at two points (Fig. 1d) or $h$ lies over $p$ and they do not intersect (Fig. 1e). In the first case, we observe a bistability regime (with two equilibria): the lower (in terms of host biomass) equilibrium E1 is stable (Fig. 2a) or unstable (Fig. 2b), depending on parameter values, and the higher equilibrium E2 is

Fig. 2 Phase planes of two stability cases for equilibrium $E 1$ from Fig. 1d. Hemiparasitic and host isoclines are marked by solid line $p$ and dotted line $h$. Dashed lines with arrows denote oriented trajectories of system (4). All initial conditions in the area bordered by the dashed line (without arrows) are converging to equilibrium E1. Initial conditions outside the dashed line bordered area lead to the host-only equilibrium $[K, 0]$. In a, $E 1$ is a stable equilibrium (parameters of Eq. 4: $e=1$; $a=0.6 ; b=6 ; d=7 ; m=0.1$; $\left.m_{1}=0.006 ; c=0.1 ; K=14\right)$. In $\mathbf{b}, E 1$ is an unstable equilibrium with a stable limit cycle around it (parameters are similar as previous, except: $b=5$; $K=15$ )
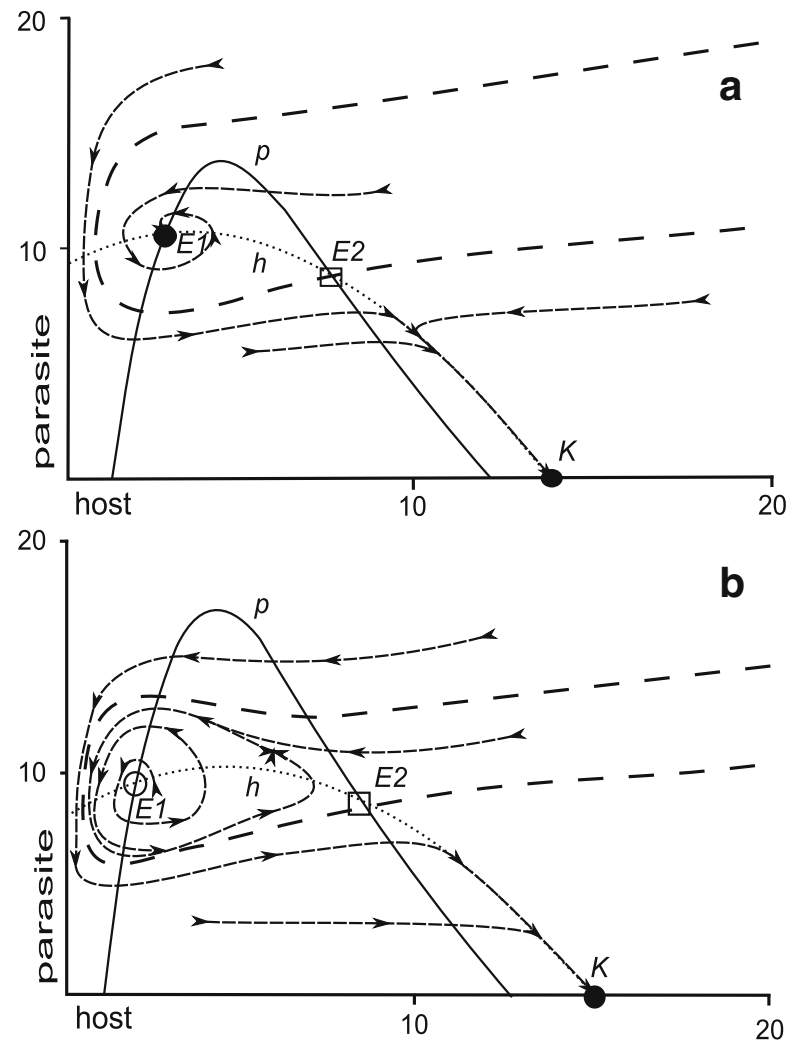
always an unstable saddle point. In the case where $E 1$ is unstable, then there is a stable limit cycle around (Fig. 2b) it or all initial conditions lead to the host-only equilibrium $[K, 0]$. With increasing $K$, the area of attraction of equilibrium $E 1$ (or the surrounding limit cycle) decreases and the area of attraction of the host-only equilibrium $[K, 0]$ increases until the host isocline moves over the hemiparasite isocline for any value of host biomass. The second case, where $h$ lies over $p$, again corresponds to only two trivial equilibria $[0,0]$ and $[K, 0]$ of which only the latter is stable, and no non-trivial equilibrium (Fig. 1e). In both of these cases, the carrying capacity of host plants is too high and hosts can thus attain a great amount of biomass, which leads to intensive shading and decline of hemiparasites. For parameter ranges in which the equilibrium E1 is stable or unstable, see Fig. 3.

\section{Carrying Capacity K Lies Inside (A,B) Range}

If $K$ lies inside the $(A, B)$ range, then we know from above that the equilibrium $[K, 0]$ is unstable (saddle point). Numerical calculations suggest that there exist one or three non-trivial equilibria at the intersections of isoclines. If there is only one intersection, then this equilibrium point is stable or unstable (Fig. 1b). If the isoclines intersect at three non-zero equilibrium points, we observe another bistability regime: the lowest (in terms of host biomass) equilibrium $E 1$ is stable or unstable, the intermediate one (E2) is always unstable (saddle point) and the highest one $(E 3)$ is again stable or unstable (Fig. 1c). For parameter ranges in which the equilibria E1 and $E 3$ are stable or unstable, see Fig. 3.

The situation where the carrying capacity $K$ lies in the $(A, B)$ range is the main area of coexistence of host and hemiparasite plants and therefore we refer to $(A, B)$ as the coexistence range. Coexistence (hemiparasites present) equilibrium appears after $K$ enters the range $(A, B)$. Hemiparasite species biomass for $K$ in the $(A, B)$ range is shown in Fig. 4. Biomass of the hemiparasite increases with a concave down shape until it exceeds the top of the hemiparasite isocline or until there appear two other equilibria; the last of them will become a new stable coexistence equilibrium. After that, biomass of the hemiparasite decreases to zero.

\section{Dependence on Parameters}

An increase in parameters $a$ and $e$, the hemiparasite maximum unit gain and efficiency of transformation of the host biomass to that of the hemiparasite, respectively, leads to an extension of the length of range $(A, B)$. This means that the hemiparasite maximum unit gain and transformation efficiency directly refer to the length of the coexistence range. An increase in parameter $b$, the host biomass necessary to achieve one-half the maximum "consumption" rate, reduces the length of the coexistence range $(A, B)$ as well as the maximum of the hemiparasite isocline. Also, if $b$ is increased, the functional response $f(x)$ will grow at a slower rate, and the left (growing) side of the hemiparasite isocline will increase less abruptly. Changing $m$, the intrinsic per capita hemiparasite mortality rate, does not change the isocline shape, but only moves the isocline along the $y$ axis up or down.

Parameters corresponding to the mechanisms that extend the RosenzweigMacArthur predator-prey model play a significant role, too. Parameter $d$, the host 

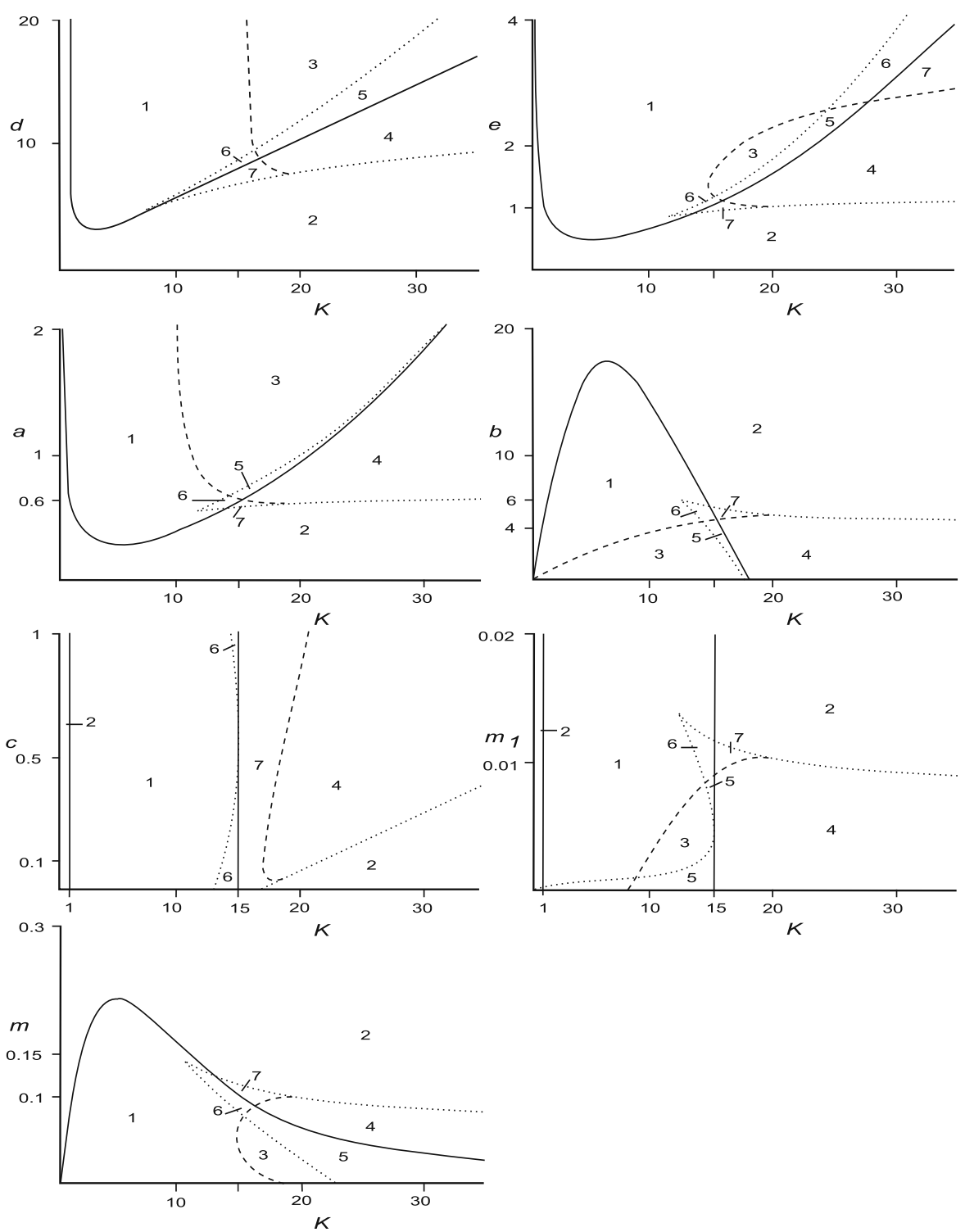

Fig. 3 Analysis of equilibria for all parameters of system (4) along the carrying capacity $K$. The solid line delimits the area in which $K$ lies in the $(A, B)$ range. The dotted line then delimits the area where the equilibrium $E 2$ exists. The dashed line with a connection to the dotted line delimits the area where the equilibrium $E 1$ is unstable and surrounded by a stable limit cycle. Numbers denote areas, bordered with lines, where model (4) has different behavior. In area 1, there is only the stable equilibrium E1 (Fig. 1b). In area 2 , there is only the stable equilibrium $[K, 0]$ (Fig. 1a and e). In area 3, there is only the non-trivial equilibrium $E 1$ which is unstable and surrounded by a stable limit cycle (Fig. 1b). In area 4, $[K, 0]$ is a stable equilibrium and $E 1$ is unstable and surrounded by a stable limit cycle (Fig. 1d). In area 5, there are three non-trivial equilibria $E 1, E 2$ and $E 3$, where $E 1$ is unstable and surrounded by a stable limit cycle, E2 is an unstable saddle point and $E 3$ is stable or unstable (Fig. 1c). Area 6 is similar to area 5, but $E 1$ is stable (Fig. 1c). In area 7, there are three equilibria $E 1, E 2$ and $[K, 0]$, where $E 1$ and $[K, 0]$ are both stable and $E 2$ is an unstable saddle point (Fig. 1d). Parameters of Eq. 4 used to draw these diagrams: $e=1 ; a=$ $0.6 ; b=5 ; d=8 ; m=0.1 ; m_{1}=0.01 ; c=0.1$ 


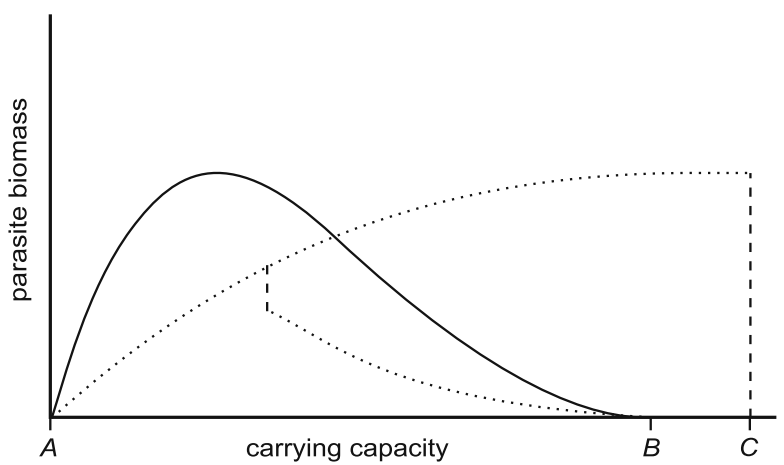

Fig. 4 Generalized graph with hemiparasites biomass along increasing productivity $K>A$. The solid line shows situations where the hemiparasite and host isoclines have only one non-trivial intersection for $K$ in the $(A, B)$ range (Fig. 1b). Parameters of Eq. 4 used to draw a similar line: $e=1 ; a=0.5 ; b=5 ; d=9 ; m=0.1$; $m_{1}=0.01 ; c=0.1$. The dotted lines correspond to situations where there is more than one non-trivial intersection with different biomass (Fig. 1c and d). Parameters used to draw similar lines are the same as the previous ones, except: $a=0.6 ; d=8$. The upper dotted line is for the $E 1$ equilibrium and the lower one for the $E 3$ equilibrium. Increasing the carrying capacity leads to a decrease in the area of attraction of point $E 1$ (or the surrounding limit cycle) and an increase in the area of attraction of point $E 3$ once $K<B$ and point $[K, 0]$ once $K>B$. The point $C \geq B$ is the carrying capacity where the host isocline surpasses the hemiparasite isocline (Fig. 1e). These two line types correspond to two alternative scenarios and hence to different parameter sets

biomass necessary to achieve one-half the maximum of the scaling function (3) due to shading, has the opposite effect to parameter $b$. Its increase extends the coexistence range, results in a higher maximum of the hemiparasite isocline and causes the right (declining) side of the hemiparasite isocline to decrease at a slower rate. If $d$ is increased, intensity of shading is reduced. An increase in parameter $c$, the competitive ability of hemiparasites for light relative to their hosts, leads to a decrease in the carrying capacity of host plants in the presence of hemiparasites. Finally, an increase in parameter $m_{l}$, the measure of negative density dependence among hemiparasites, also decreases the maximum value of the hemiparasite isocline.

For parameter ranges with respect to the host carrying capacity $K$ that lead to the existence of any system equilibrium, see Fig. 3. From this figure we observe that there is only one relatively restricted area 3 where the system has a unique nontrivial equilibrium $E 1$ that is unstable and surrounded by a stable limit cycle. Changes in unconstrained parameters $d$ and $a$ might predominantly take the system (4) to area 3. In area 5, E1 is unstable and $E 3$ is a stable or unstable equilibrium (for the parameter values used to create Fig. 3, E3 was always stable). In all the other areas, there always exist one or two non-trivial stable equilibria.

Extensions of the Rosenzweig-MacArthur predator-prey model guide the different dynamics; see Fig. 5 for examples. Removal of the competitive effect of parasite on the host by shading (parameter $c$ ) leads to a decrease of the area where initial conditions drive the system (4) to coexistence of species; compare Fig. 2a and Fig. 5a. Removal of intra-specific parasitism (parameter $m_{l}$ ) has the opposite effect. This change also destabilizes equilibrium $E 1$ and a stable limit cycle appears around E1; compare Fig. 2a and 5b. After removal of shading of hemiparasites by their hosts (function $g(x)$ ), the behavior of the system distinctively changes (see Fig. 5c). 
Fig. 5 Phase planes of system (4) without extension of the Rosezweig-MacArthur model (base scenario is Fig. 2a). Hemiparasitic and host plant isoclines are marked by solid line(s) $p$ and dotted line $h$. Dashed lines with arrows denote oriented trajectories of the system (4). All initial conditions in the area bordered by the dashed line (without arrows) converge to equilibrium E1. Initial conditions outside the dashed line bordered area lead to the host only equilibrium $[K, 0]$. Parameters of Eq. 4 used to draw these diagrams: $e=1 ; a=0.6 ; b=6 ; d=7$; $m=0.1 ; m_{1}=0.006 ; c=0.1$; $K=14$. Phase planes a and b reflect the scenarios of system (4) where $c=0$ and $m_{1}=0$. Phase plane c shows a situation where the hemiparasitic equation is without the $g(x)$ function
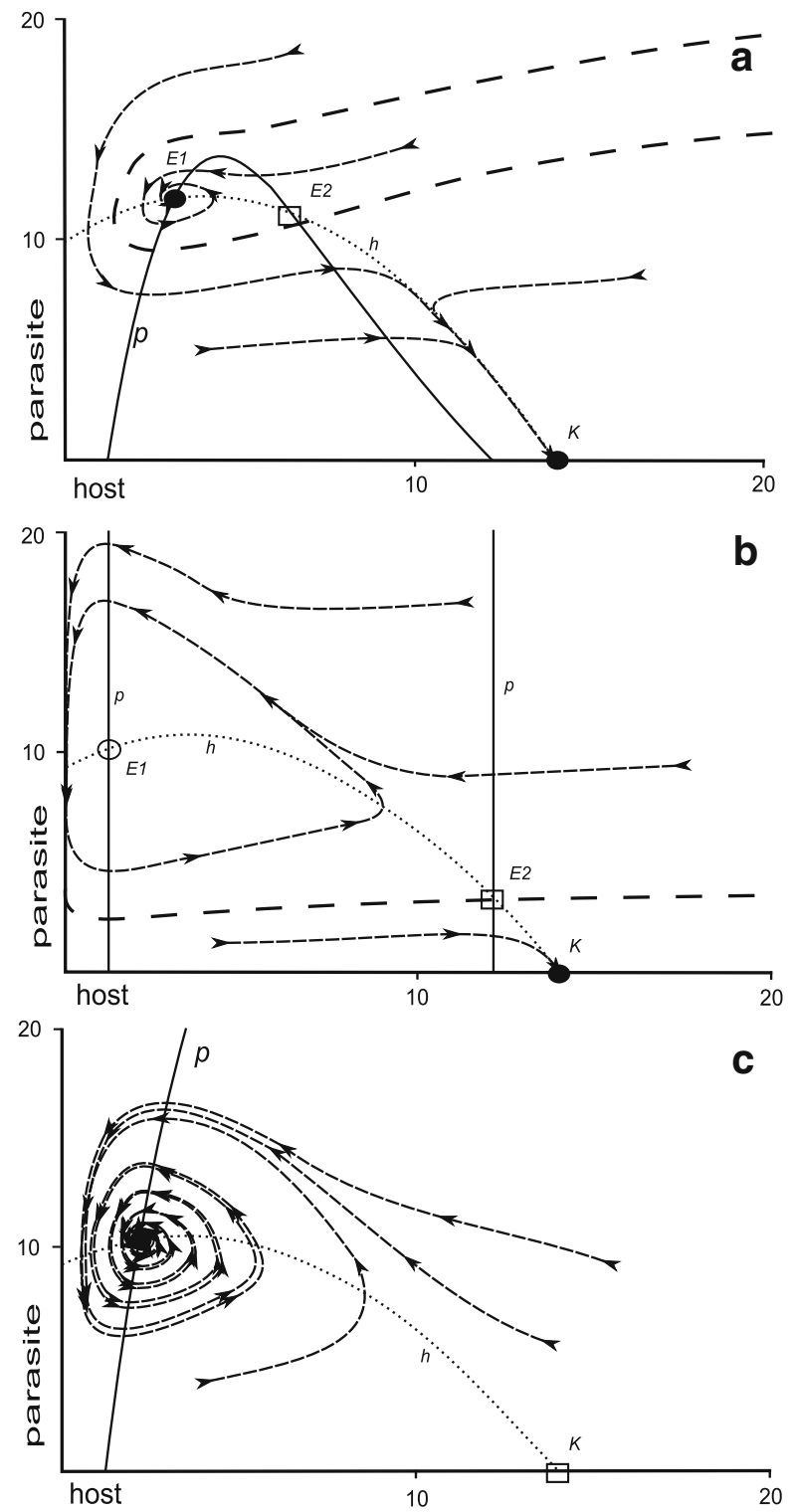

Except at very low productivities, there is always some coexistence equilibrium (stable or unstable with a stable limit cycle around). Also extinction of both species could occur with starting conditions having a great amount of hemiparasites and significantly smaller amount of host plants even at high productivities. Both previous situations are not realistic and are not observed in nature (van Hulst et al. 1987; Matthies 1995). Overall, the addition of a function describing shading of hemiparasites by their hosts is the most important amendment leading to realistic behavior of the system. Shading of hosts by hemiparasites and intra-specific parasitism also stabilize the system but their effect is fairly less pronounced. 


\section{Comparison with Field Data}

To check whether our prediction of the change in the biomass of hemiparasites along the productivity gradient (Fig. 4) can help explain and understand observed empirical patterns, we re-analyzed the data collected by Hadač (1969) on biomass and species composition in natural alpine communities in the Belianske Tatry Mts. (Slovakia). Hadač (1969) characterized each community type $(n=13)$ by total dry biomass (which we take as a surrogate for productivity) from five relevés (relevé $=$ list of all species per plot with ocular estimation of percent cover). In our analysis we excluded one community type dominated by a small shrub, Vaccinium uliginosum L.; its biomass was built by its perennial woody stem, which may not reflect annual productivity. Both the total number of hemiparasitic plant species and their proportion (percentage of parasitic plants among all plant species in the five relevés) decreased with increasing biomass in the community; the hemiparasites were rare or even absent in highly productive plant communities (Fig. 6, only the proportion shown). This phenomenon is well predicted by our model.

\section{Discussion}

We propose a model for the interactions between hemiparasitic and host plants that extends the Rosenzweig-MacArthur predator-prey model (Berryman 1992; Kot 2001; Pastor 2008) and considers intra-specific interactions among hemiparasites and competition of both plant species for light. The intra-specific interactions include parasitism between hemiparasites and mutual shading (Prati et al. 1997; Matthies 2003). The importance of competition for light grows with increasing productivity of the environment (Wilson and Tilman 1993; Lepš 1999). Our model is designed to describe obligate hemiparasites, the most common type in this group (Press 1989; Press and Phoenix 2005). This leads to the special form of hemiparasite equation that is host oriented and not directly environmental-productivity oriented.

Fig. 6 Relationship between the proportion of hemiparasitic plants (percentage of total plant species that were parasitic in the five relevés) and the biomass of vascular plants (with hemiparasites) in alpine meadow communities, based on data by Hadač (1969). The regression line was fitted using generalized linear models, and the equation is percentage of hemiparasitic species $=\exp$ (2.10-0.003566 $\times$ biomass $\left.\left[\mathrm{g} / \mathrm{m}^{2}\right]\right), R^{2}=0.4444, P=0.013$

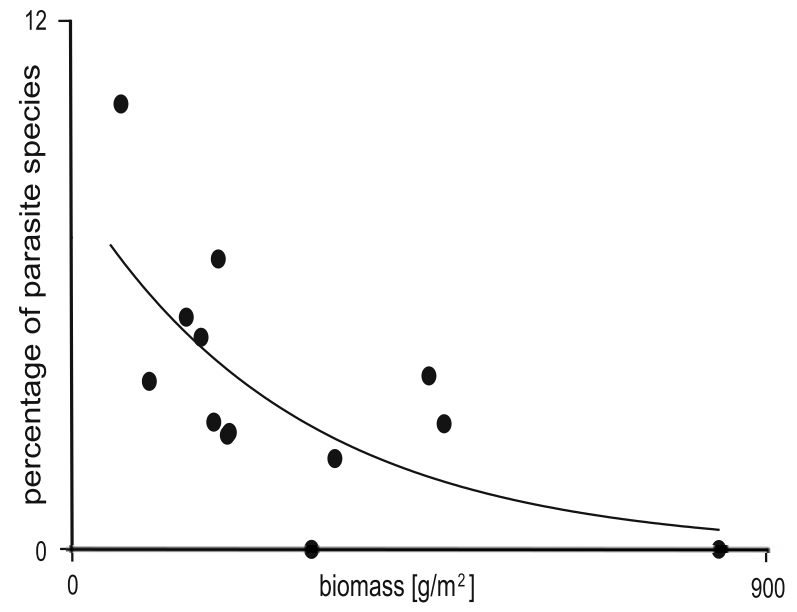


Our extended model, compared with the Rosenzweig-MacArthur predator-prey model, includes several additional processes (light competition between host types and intra-specific interactions among hemiparasites) and results in more complex dynamical behavior (occurrence of two types of bistability). Generally, the new parameters corresponding to the novel mechanisms help stabilize system dynamics (Fig. 3). However, there are still parameter ranges that give rise to unstable dynamics, that is, result in hemiparasite extinction, similarly to Cameron et al. (2009). Adding a shading function to the Rosenzweig-MacArthur model was the most useful in explaining the relation between hemiparasites and host plants.

We analyzed the model along the productivity gradient of the environment, which we identified with the carrying capacity of the environment for the host plant species. In poor productivity environments, host biomass is too low to sustain hemiparasites, which eventually go extinct (Fig. 1a); this corresponds to the results of Westbury and Dunnett (2007). An initial increase in productivity makes coexistence between hosts and hemiparasites possible (Fig. 1b,c and d). There is also a range of low environmental productivities in which the biomass of hemiparasites grows (Fig. 4). This situation continues until a new coexistence equilibrium (E3) appears or the host isocline surpasses the peak of hemiparasite isocline. Any further increase in productivity leads to a slower decrease in the proportion of hemiparasite biomass or its sudden decline, as suggested by Westbury and Dunnett (2007). That decrease continues to zero until we reach the end of the coexistence range. If we further increase productivity, the carrying capacity of the host plants becomes too high and therefore hosts could gain a greater amount of biomass, which leads to intensive shading and decline and eventual extinction of hemiparasites (Fig. 1d and e), as suggested by van Hulst et al. (1987). Cameron et al. (2005) similarly stated that there is a significant interaction between nutrients and parasitism. If the shading of a parasite by the host was excluded from the model, the competitive exclusion of the parasite population under high productivity disappeared. This corresponds to the behavior of holoparasites, which do not need light, and are known to be able to grow also in highly productive communities (e.g., some species of Orobanche).

The length of the coexistence range for hemiparasites and host plants could be extended by increasing the maximum hemiparasite unit gain or efficiency of transformation of host biomass to that of the hemiparasite; an opposite effect is due to an increase of the half-saturation parameter of the hemiparasite unit gain. An increase of the half-saturation parameter corresponding to shade-tolerance leads to the extension of the coexistence range.

Our model results thus confirm the conjecture of Matthies (1995) that hemiparasites are restricted to nutrient-poor habitats, because of a balance between the effects of parasitism and light competition. Our predictions are also in agreement with the data of Hadač (1969), showing that increasing the total biomass of plants leads to a decrease in the percentage of hemiparasitic plants (Fig. 6), and with our own field experience. Similarly, fertilizing and higher host community biomass lead to decreased seedling survival (Mudrák and Lepš 2010), decreased fruiting (van Hulst et al. 1987) and density of hemiparasites (Westbury and Dunnett 2007).

The model of Cameron et al. (2009) is designed to describe a three-species community (hemiparasites, grasses and forbs) at two levels of nutrients (low and 
high). Whereas stable dynamics emerge under low-nutrient levels, in high-nutrient treatments stable dynamics appear only after accounting for spatial effects (local rather than global competition). The former prediction is equal to ours: coexistence of hemiparasites and their hosts is possible under low-nutrient levels. Our model predicts extinction of hemiparasites under high-nutrient levels. This corresponds to the greater parameter area of unstable and no hemiparasites dynamics in the nonspatial Cameron et al. model under high nutrient levels than low levels.

Another model used to describe the host-hemiparasite interaction is that by Smith (2000), the results of which are opposite to ours. Namely, this model predicts that, with increasing productivity, the proportion of biomass of hemiparasites will also increase. These contrasting results come from the different model assumptions. In fact, the model of Smith (2000) is oversimplified, as it covers neither the intraspecific interactions among hemiparasites nor aboveground competition for light. The latter is especially important in high-productivity environments (Grime 1979; Wilson and Tilman 1993; Lepš 1999). Because hemiparasites are quite poor in competition for light with their hosts (van Hulst et al. 1987; Matthies 1995; Svensson and Carlsson 2004) and have high light requirements (ter Borg 1985), they usually lose in competition in productive environments. However, Hwangbo and Seel (2002) suggest that light availability does not affect the growth of Rhinanthus minor L. Also, it has been proposed that, after establishment, sward density is not important for the persistence of Rhinanthus (de Hullu et al. 1985). On the contrary, Mudrák and Lepš (2010) found that the mortality of Rhinanthus minor L. was higher in fertilized (and so more productive) plots. Unlike in the model by Smith (2000), it is not possible for hemiparasites to outcompete the host species (neither in reality, nor in our model).

Hemiparasites are considered as ecosystem engineers, because they alter the surrounding physical environment and can increase community diversity (Press and Phoenix 2005; Westbury and Dunnett 2007). Hemiparasites influence the structure and functioning of the plant community (Joshi et al. 2000; Press and Phoenix 2005; Cameron et al. 2009). For example, Rhinanthus has been suggested as a tool in the restoration of species-rich meadows, because it suppresses grasses (preferred hosts) in favor of forbs (Westbury and Davies 2005; Cameron et al. 2006; Ameloot et al. 2006; Westbury and Dunnett 2007). Our results suggest that the function of ecosystem engineers via suppression of preferred host species is possible only in nutrient-poor habitats, due to light availability, while in nutrient-rich habitats they will have only a small and temporary effect.

Our model is based on realistic assumptions about the host-hemiparasite relationship, namely that the relationship is parasitic belowground where the amount of host roots limits the growth of hemiparasites. Aboveground, limited light availability governs both the density dependence of the host population and the competition between hosts and their hemiparasites. Finally, the hosts are better competitors for light than the hemiparasites. Based on these assumptions, the model predicts that the stable coexistence of host and hemiparasite niche is limited to intermediate productivities. At low productivities, the hemiparasite is not able to survive due to limitation of host availability; however, with increasing efficiency of parasitism, the environments with decreasing productivities can support the hemiparasite. At high productivity levels, the hemiparasite is outcompeted by its host. 
This decline, which is in good agreement with our field experience, is documented in the literature, and can be supported by published data.

In his classic paper, Levins (1966) noted the trade-off between generality, precision, and realism of population ecology models. Our model is an attempt to capture the most general features of the hemiparasite - host system. We have demonstrated that, should the model realistically mimic the pattern of changes in system behavior on a productivity gradient, aboveground competition for light between the host and hemiparasite must be included. To keep the model general, we had to use many simplifications. Similarly to other general models (including the one of Smith 2000), we have used a system of differential (not difference) equations, because they are better resoluble analytically. To keep the model simple and general, the size of each component population is characterized by a single number (biomass). Consequently, the changes in population density cannot be distinguished from changes in the size of individuals. We tacitly assume that both the competitive effects and amount of water and nutrients available for parasitism are determined by biomass itself. These simplifications are acceptable if all of the species are in the same layer; consequently, the model is suited for herbaceous vegetation. If we included woody hosts or woody root hemiparasites (e.g., Santalum), the situation would have been more complicated; in particular, biomass would not be a good measure of either resources available, or the effect of populations. Consequently, the field data used for comparison come also from herbaceous vegetation.

More realistic models would undoubtedly include differential effects of population density and size of individuals (together with inclusion of the effect of host and hemiparasite individuals' height), and also seasonal changes in growth rate, rate of parasitism and mortality. In particular, mortality (defined as death of individual plants) is a highly seasonal phenomenon, and depends on the life history of individual species - e.g., all the annuals die at the end of the season. As our model does not reflect the seasonality, for the annuals, we have to assume the standing biomass of the hemiparasite at the height of the growing season in one year is converted, through the production of seeds and seedlings, in an identical hemiparasite biomass at the beginning of the next growing season. Because of differences in life-histories of individual species, models reflecting seasonality would necessarily be less general; they would probably need to be species specific, or focused on functionally homogeneous groups of species.

Acknowledgments We are grateful for helpful discussions with Jakub Těšitel and Martina Petrů. We highly appreciate comments of two anonymous reviewers, which greatly improved the paper. We thank Keith Edwards for linguistic improvement of our manuscript. The research was supported by grants GAČR 206/08/H044, GAAV IAA601410805, MSM6007665801 and Z50070508. Access to the MetaCentrum computing facilities provided under the research grant MSM6383917201 is highly appreciated.

\section{References}

Abrams PA, Ginzburg LR (2000) The nature of predation: prey dependent, ratio dependent or neither? Trends Ecol Evol 15:337-341 
Ahonen R, Pustinen S, Mutikainen P (2006) Host use of a hemiparasitic plant: no trade-offs in performance on different hosts. J Evol Biol 19:513-52

Ameloot E, Verheyen K, Bakker JP, De Vries Y, Hermy M (2006) Long-term dynamics of the hemiparasite Rhinanthus angustifolius and its relationship with vegetation structure. J Veg Sci 17:637-646

Berryman AA (1992) The origins and evolution of predator prey theory. Ecology 73:1530-1535

Cameron DD, Hwangbo JK, Keith AM, Geniez JM, Kraushaar D, Rowntree J, Seel WE (2005) Interactions between the hemiparasitic angiosperm Rhinanthus minor and its hosts: from the cell to the ecosystem. Folia Geobot 40:217-229

Cameron DD, Coats AM, Seel WE (2006) Differential resistance among host and non-host species underlies the variable success of the hemi-parasitic plant Rhinanthus minor. Ann Bot (Oxford) 98:1289-1299

Cameron DD, Geniez JM, Seel WE, Irving LJ (2008) Suppression of host photosynthesis by the parasitic plant Rhinanthus minor. Ann Bot (Oxford) 101:573-578

Cameron DD, White A, Antonovics J (2009) Parasite-grass-forb interactions and rock-paper-scissor dynamics: predicting the effects of the parasitic plant Rhinanthus minor on host plant communities. $J$ Ecol 97:1311-1319

de Hullu E, Brouwer T, ter Borg S (1985) Analysis of the demography of Rhinanthus angustifolius populations. Acta Bot Neerl 34:5-22

Dhooge A, Govaerts W, Kuznetsov YA (2003) MATCONT: A Matlab package for numerical bifurcation analysis of ODEs. ACM Trans Math Software (TOMS) 29:141-164

Ellner S, Guckenheimer J (2006) Dynamic models in biology. Princeton University Press, Princeton, NJ

Freedman HI, Wolkowicz GSK (1986) Predator-prey systems with group defense: the paradox of enrichment revised. Bull Math Biol 48:493-508

Grime JP (1979) Plant strategies and vegetation processes. Wiley, New York

Hadač E (1969) Die Pflanzengesellshaften des Tales “Dolina Siedmich prameňov" in der Belaer Tatra. Vydavatel'stvo Slovenskej Akadémie Vied, Bratislava

Hwangbo JK, Seel WE (2002) Effects of light availability on attached Rhinanthus minor (L.), an angiospermatic root hemiparasite. $J$ Pl Biol 45:10-106

Joshi J, Matthies D, Schmid B (2000) Root hemiparasites and plant diversity in experimental grassland communities. J Ecol 88:643-644

Kot M (2001) Elements of mathematical ecology. Cambridge University Press, Cambridge

Kuang Y (2004) Basic properties of mathematical population models. Biomathematics 17:129-142

Lepš J (1999) Nutrient status and disturbances: an experimental test of relationships in a wet meadow. $J$ Veg Sci 10:219-230

Levins R (1966) The strategy of model building in population biology. Amer Sci 54:421-431

Matthies D (1995) Parasitic and competitive interactions between the hemiparasites Rhinanthus serotinus and Odontites rubra and their host Medicago sativa. J Ecol 83:245-251

Matthies D (1996) Interactions between the root hemiparasite Melampyrum arvense and mixtures of hosts plants: heterotrophic benefit and parasite-mediated competition. Oikos 75:118-124

Matthies D (2003) Positive and negative interactions among individuals of a root hemiparasite. Plant Biology 5:79-84

Mudrák O, Lepš J (2010) Interactions of Rhinanthus minor L. with its host plant community at two nutrient levels. Folia Geobot 45 (this issue) doi:10.1007/s12224-010-9078-1

Pastor J (2008) Mathematical ecology of population and ecosystems. Wiley-Blackwell, Chichester

Petrů M, Lepš J (2000) Regeneration dynamics in populations of two hemiparasitic Pedicularis species in wet grasslands. In White PS, Mucina L, Lepš J (eds) Vegetation science in retrospect and prospective. Opulus Press, Uppsala, pp 329-333

Polking J, Arnold D (1999) Ordinary differential equations using MATLAB. Prentice Hall, Upper Saddle River, NJ

Prati D, Matthies D, Schmid B (1997) Reciprocal parasitation in Rhinanthus serotinus: a model system of physiological interaction in clonal plants. Oikos 78:221-229

Press MC (1989) Autotrophy and heterotrophy in root hemiparasites. Trends Ecol Evol 4:258-263

Press MC, Phoenix GK (2005) Impacts of parasitic plants on natural communities, New Phytol 166:737751

Puustinen S, Salonen V (1999) Effects of intensity and duration of infection by a hemiparasitic plant, Rhinanthus serotinus, on growth and reproduction of a perennial grass, Agrostis capillaris. Ecography 22:160-168

Seel WE, Press MC (1996) Effect of repeated parasitism by Rhinanthus minor on the growth and phohosynthesis of perennial grass Poa alpina. New Phytol 134:495-502 
Smith D (2000) The population dynamics and community ecology of root hemiparasitic plants. Amer Naturalist 155:13-23

Svensson BM, Carlsson BA (2004) Significance of time of attachment, host type, and neighbouring hemiparasites in determining fitness in two endangered grassland hemiparasites. Ann Bot Fenn 41:6375

ter Borg SJ (1985) Population biology and habitat relations of some hemiparasitic Scrophulariaceae. In White J (ed) The population structure of vegetation. Dr. W. Junk Publishers, Dordrecht, pp 463-487

Tilman D (1988) Dynamics and structure of plant communities. Princeton University Press, Princeton

van Hulst R, Shipley B, Thériault A (1987) Why is Rhinanthus minor (Scrophulariaceae) such a good invader? Canad J Bot 65:2373-2379

Westbury DB, Davies A (2005) Yellow rattle - its natural history and use in grassland restoration. British Wildlife 17:93-98

Westbury DB, Dunnett NP (2007) The impact of Rhinanthus minor in newly established meadows on a productive site. Appl Veg Sci 10:121-129

Wilson SD, Tilman D (1993) Plant competition and resource availability in response to disturbance and fertilization. Ecology 74:599-611

Received: 29 January 2009 / Revised: 8 January 2010 / Accepted: 28 January 2010 /

Published online: 21 August 2010

\section{Appendix 1}

Jacobian of model (4) is as follows

$$
\mathbf{J}\left[\begin{array}{l}
x \\
y
\end{array}\right]=\left(\begin{array}{ll}
a 11 & a 12 \\
a 21 & a 22
\end{array}\right)
$$

where

$$
\begin{aligned}
& a 11=1-\frac{c y+x}{K}+x\left(\frac{a y}{(x+b)^{2}}-\frac{1}{K}\right)-\frac{a y}{x+b} \\
& a 12=-x\left(\frac{a}{(x+b)}+\frac{c}{K}\right) \\
& a 21=\left(\frac{a d^{2} e}{(x+b)\left(x^{2}+d^{2}\right)}-\frac{a d^{2} e x}{(x+b)^{2}\left(x^{2}+d^{2}\right)}-\frac{2 a d^{2} e x^{2}}{(x+b)\left(x^{2}+d^{2}\right)^{2}}\right) y \\
& a 22=-2 y m_{1}+\frac{a d^{2} e x}{(x+b)\left(x^{2}+d^{2}\right)}-m
\end{aligned}
$$

a11 (a12) is the partial derivative of the right-hand side of the host equation of model (4) according to $x(y)$ and $a 21$ (a22) is the partial derivative of the right-hand side of the hemiparasite equation of model (4) according to $x(y)$. 